\title{
Arbitragegewinne durch Optimierung der betrieblichen Finanzierung
}

\author{
Teil 2: Lösung
}

\author{
Ernst Troßmann und Manuel Kallabis
}

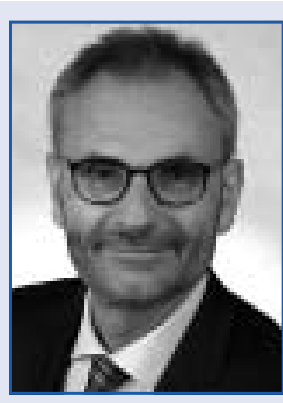

Prof. Dr. Ernst Troßmann ist Inhaber des Lehrstuhls Controlling im Institut für Financial Management der Universität Hohenheim. Bevorzugte Forschungsgebiete: Instrumente des Controlling, entscheidungsorientiertes Rechnungswesen, Investitionsrechnung.

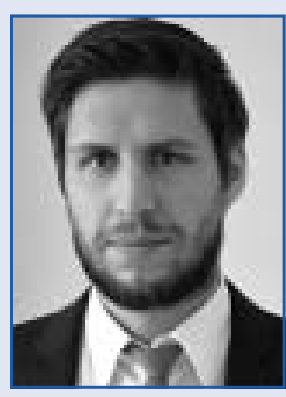

Dipl. oec. Manuel Kallabis ist wissenschaftlicher Mitarbeiter am Lehrstuhl Controlling im Institut für Financial Management der Universität Hohenheim. Bevorzugte Forschungsgebiete: Lebenszyklusrechnung, Investitionsrechnung, entscheidungsorientiertes Rechnungswesen.

Wenn sich neuer Finanzbedarf auf verschiedene Weise decken lässt, kann auch eine Änderung der bisherigen Finanzierung vorteilhaft sein. Dann ist durch Umfinanzierung (z. B. Umschuldung) ein Arbitragegewinn möglich. Wie dies systematisch geprüft und wie schrittweise umfinanziert wird, behandelt diese Fallstudie. Fallbeschreibung und Aufgaben (Teil 1 der Fallstudie) können in WiSt-Heft, Nr. 4/2016, S. 217-218, nachgelesen werden.

Stichwörter: Arbitragegewinn, betriebliche Finanzierungssituation, verallgemeinerte Marktzinsmethode, Umschuldung, Unternehmenskauf

Die Lösungen der Aufgaben stehen als Excel-Tabellen auf der WiSt-Homepage unter www.beck-shop.de/ccb zum Download bereit.

\section{Aufgabe la)}

In $A b b .1$ ist die Finanzierungssituation der Blitz AG (Finanzierung A) bis zum Jahr 4 dargestellt. Die Kredite können ausgeweitet oder auch zurückgezahlt werden. Der ma- ximale Erhöhungsbetrag ist als Obergrenze, der maximale Tilgungsbetrag als Untergrenze angegeben.

Die Untergrenze von Kredit $\mathrm{A}_{3}$ ergibt sich als Differenz aus der ursprünglichen Kreditsumme in Höhe von 1,1 Mio. $€$ und den zwei bereits bezahlten Raten in Höhe von jeweils $220.000 €$. Für Kredit $\mathrm{A}_{4}$ berechnet man die Restschuld (und somit die maximale Reduzierung), indem man die Annuität des ersten Jahres in einen Zins- und einen Tilgungsanteil aufspaltet und den errechneten Tilgungsanteil von der ursprünglichen Kreditsumme subtrahiert.

\section{Aufgabe 1b)}

Die auf einen Kreditbereitstellungsbetrag von 1,00 € normierten Zahlungsverläufe der einzelnen Kredite der Finanzierung A sind spaltenweise in $A b b .2$ aufgeführt.

\section{Aufgabe 2a)}

Die Finanzierungssituation der Blank GmbH (Finanzierung B) ist in $A b b .3$ zusammengestellt. Die normierten Zahlungsströme der zugehörigen Finanzgeschäfte $B_{1}$ bis $\mathrm{B}_{4}$ zeigt $A b b .4$.

\section{Aufgabe 2b)}

Nach einer Übernahme der Blank GmbH durch die Blitz AG würden für die nächsten vier Jahre insgesamt acht (unabhängige) Finanzgeschäfte existieren, mit denen die vier Jahres-Zahlungssalden ausgeglichen werden können. Damit gibt es für diesen Ausgleich keine eindeutige Lösung. Vielmehr kann unter den bestehenden Geschäften gewählt und damit optimiert werden. Angesichts dessen ist zu prüfen, inwieweit finanziell ungünstigere Finanzgeschäfte durch günstigere Geschäfte ersetzt werden können. Um die Vorteilhaftigkeit einer Erhöhung oder Verringerung einzelner Kredit- oder Anlagegeschäfte bewerten zu können, ist zunächst eine eindeutige finanzielle Ausgangslage festzulegen, als Standardfinanzierung bezeichnet. Für den hier beschriebenen Betrachtungszeitraum besteht sie aus vier Finanzierungsgeschäften, mit denen neu zu beurteilende Projekte über die nächsten vier Jahre finanziert werden würden. Gegenüber den Krediten und Anlagen der 


\begin{tabular}{|c|c|c|c|c|c|}
\hline \multicolumn{6}{|c|}{ Finanzierung $\mathbf{A}$} \\
\hline $\begin{array}{l}\text { Kredit-l } \\
\text { Anlage- } \\
\text { geschäft }\end{array}$ & Kredit-/Anlagetyp & $\begin{array}{l}\text { Rest- } \\
\text { laufzeit }\end{array}$ & Zinssatz & $\begin{array}{c}\text { maximale } \\
\text { Reduzierung: } \\
\text { Untergrenze } \\
\mathrm{B}_{\mathrm{i}}^{\mathrm{u}}\end{array}$ & $\begin{array}{c}\text { maximale } \\
\text { Erhöhung: } \\
\text { Obergrenze } \\
\mathbf{B}_{\mathbf{i}}^{\circ}\end{array}$ \\
\hline$A_{1}$ & $\begin{array}{l}\text { Endfälliger Kassakredit mit } \\
\text { jährlicher Zwischenzinszahlung }\end{array}$ & $1 \mathrm{Jahr}$ & $3,4 \%$ & $-440.000 €$ & $560.000 €$ \\
\hline$A_{2}$ & $\begin{array}{l}\text { Endfälliger Kassakredit mit } \\
\text { jährlicher Zwischenzinszahlung }\end{array}$ & 2 Jahre & $5,5 \%$ & $-350.000 €$ & $650.000 €$ \\
\hline$A_{3}$ & Ratenkredit & 3 Jahre & $5,7 \%$ & $-660.000 €$ & $800.000 €$ \\
\hline $\mathbf{A}_{4}$ & Annuitätendarlehen & 4 Jahre & $7,2 \%$ & $-620.101 €$ & $800.000 €$ \\
\hline
\end{tabular}

Abb. 1: Detailangaben zu den Finanzierungsmöglichkeiten durch Variation der bestehenden Kredite der Blitz AG

\begin{tabular}{|c|c|c|c|c|}
\hline $\begin{array}{c}\text { Kredit-I } \\
\text { Anlage- } \\
\text { geschäft }\end{array}$ & $\mathbf{A}_{1}$ & $\mathbf{A}_{2}$ & $\mathbf{A}_{3}$ & $\mathbf{A}_{4}$ \\
\hline Jahr t & $1,00000 €$ & $1,00000 €$ & $1,00000 €$ & $1,00000 €$ \\
\hline 0 & $-1,03400 €$ & $-0,05500 €$ & $-0,39033 €$ & $-0,29656 €$ \\
\hline 1 & - & $-1,05500 €$ & $-0,37133 €$ & $-0,29656 €$ \\
\hline 2 & - & - & $-0,35233 €$ & $-0,29656 €$ \\
\hline 3 & - & - & - & $-0,29656 €$ \\
\hline 4 & & & &
\end{tabular}

Abb. 2: Zahlungsverläufe der vier A-Kredite

\begin{tabular}{|c|c|c|c|c|c|}
\hline \multicolumn{6}{|c|}{ Finanzierung B } \\
\hline $\begin{array}{l}\text { Kredit-/ } \\
\text { Anlage- } \\
\text { geschäft }\end{array}$ & Kredit-/Anlagetyp & $\begin{array}{l}\text { Rest- } \\
\text { laufzeit }\end{array}$ & Zinssatz & $\begin{array}{c}\text { maximale } \\
\text { Reduzierung: } \\
\text { Untergrenze } \\
\mathrm{B}_{\mathrm{i}}^{\mathrm{u}}\end{array}$ & $\begin{array}{c}\text { maximale } \\
\text { Erhöhung: } \\
\text { Obergrenze } \\
\mathbf{B}_{\mathrm{i}}^{\circ}\end{array}$ \\
\hline $\mathbf{B}_{1}$ & Anlage & 1 Jahr & $1,90 \%$ & $-75.000 €$ & unbegrenzt \\
\hline $\mathrm{B}_{2}$ & $\begin{array}{l}\text { Endfälliger Kassakredit mit } \\
\text { jährlicher Zwischenzinszahlung }\end{array}$ & 2 Jahre & $7,10 \%$ & $-580.000 €$ & $600.000 €$ \\
\hline $\mathbf{B}_{3}$ & Annuitätendarlehen & 3 Jahre & $6,70 \%$ & $-1.200 .000 €$ & $0 €$ \\
\hline $\mathbf{B}_{4}$ & Ratenkredit & 4 Jahre & $7,50 \%$ & $-850.000 €$ & $600.000 €$ \\
\hline
\end{tabular}

Abb. 3: Detailangaben zu den Finanzierungsmöglichkeiten durch Variation der Kredite der Blank GmbH

Standardfinanzierung sind auch die verbleibenden Kreditund Anlagegeschäfte zu bewerten.

Im vorliegenden Fall kann die Standardfinanzierung sowohl ausschließlich aus den Finanzgeschäften der Blitz AG als auch ausschließlich aus den Geschäften der Blank $\mathrm{GmbH}$, aber auch aus einer Mischung dieser Geschäfte gebildet werden. Da die Bewertung durch die Blitz AG erfolgt, liegt es nahe, in der Ausgangssituation deren Finanzierung A als Standardfinanzierung zu definieren. Alle anderen Finanzierungsgeschäfte, insbesondere die ursprüng- lich von der Blank GmbH stammenden, sind dann im Vergleich zur Standardfinanzierung zu bewerten: Erweisen sie sich als ungünstig, sind sie zu verringern, sind sie günstig, sind sie nach Möglichkeit auszubauen. Dazu ist jeweils der Kapitalwert der alternativen Geschäfte auf der Basis einer Finanzierung mit den Geschäften der Standardfinanzierung zu berechnen. Für jeden Kredit und jede Geldanlage der Blank GmbH sind also die zugehörigen Zahlungsverläufe aus Abb. 4 mit den Abzinsungsfaktoren der Standardfinanzierung abzuzinsen. 


\begin{tabular}{|c|c|c|c|c|}
\hline $\begin{array}{c}\text { Kredit-I } \\
\text { Anlage- } \\
\text { geschäft }\end{array}$ & $\mathbf{B}_{1}$ & $\mathbf{B}_{2}$ & $\mathbf{B}_{3}$ & $\mathbf{B}_{4}$ \\
\hline Jahr $\mathbf{t}$ & $-1,00000 €$ & $1,00000 €$ & $1,00000 €$ & $1,00000 €$ \\
\hline 0 & $1,01900 €$ & $-0,07100 €$ & $-0,37896 €$ & $-0,07500 €$ \\
\hline 1 & - & $-1,07100 €$ & $-0,37896 €$ & $-0,37500 €$ \\
\hline 2 & - & - & $-0,37896 €$ & $-0,35250 €$ \\
\hline 3 & - & - & - & $-0,43000 €$ \\
\hline 4 & - & - &
\end{tabular}

Abb. 4: Zahlungsverläufe der vier B-Finanzgeschäfte

Zunächst stellen wir die Abzinsungsfaktoren der Standardfinanzierung bereit. Dazu bildet man die Matrix A der Rückzahlungskoeffizienten sowie den Barzahlungsvektor $\mathrm{a}^{(0)}$. Sie lauten nach den Angaben aus $A b b .2$ :

$$
\begin{aligned}
& a^{(0)}=\left(\begin{array}{llll}
1 & 1 & 1 & 1
\end{array}\right) \\
& A=\left(\begin{array}{cccc}
1,03400 & 0,05500 & 0,39033 & 0,29656 \\
0 & 1,05500 & 0,37133 & 0,29656 \\
0 & 0 & 0,35233 & 0,29656 \\
0 & 0 & 0 & 0,29656
\end{array}\right)
\end{aligned}
$$

Die zur Berechnung der Abzinsungsfaktoren erforderliche Inverse der Matrix A berechnet man zu:

$$
A^{-1}=\left(\begin{array}{ccrr}
0,96712 & -0,05042 & -1,01829 & 0,10159 \\
0 & 0,94787 & -0,99898 & 0,05111 \\
0 & 0 & 2,83822 & -2,83822 \\
0 & 0 & 0 & 3,37197
\end{array}\right)
$$

Die erforderlichen Abzinsungsfaktoren erhält man aus dem Produkt $\mathrm{a}^{(0)^{2}} \cdot \mathrm{A}^{-1}$ als:

$$
z^{\prime}=\left(\begin{array}{llll}
0,96712 & 0,89745 & 0,82095 & 0,68645
\end{array}\right)
$$

Mit diesen Abzinsungsfaktoren lassen sich nun die Kapitalwerte der Zahlungsströme der B-Geschäfte ermitteln. Dies zeigt $A b b .5$.

Bei einem positiven Kapitalwert ist das Finanzgeschäft zu erhöhen, bei einem negativen Kapitalwert zu reduzieren.
Aufgrund der betragsmäßigen Größenordnung wird zunächst der Kredit $B_{2}$ betrachtet. Es ist finanziell vorteilhaft, dieses Geschäft zu reduzieren, d. h. diesen Kredit zurückzuzahlen. Mit der inversen Matrix $\mathrm{A}^{-1}$ lässt sich berechnen, wie die (bisherigen) Standardfinanzgeschäfte anzupassen sind, um die Rückzahlung von $\mathrm{B}_{2}$ zu ermöglichen (vgl. zur Methode Troßmann, 2013, S. 177 ff.):

$\left(\begin{array}{l}x_{1} \\ x_{2} \\ x_{3} \\ x_{4}\end{array}\right)=-\left(\begin{array}{cccr}0,96712 & -0,05042 & -1,01829 & 0,10159 \\ 0 & 0,94787 & -0,99898 & 0,05111 \\ 0 & 0 & 2,83822 & -2,83822 \\ 0 & 0 & 0 & 3,37197\end{array}\right) \cdot\left(\begin{array}{c}0,07100 \\ 1,07100 \\ 0 \\ 0\end{array}\right) y_{2}=\left(\begin{array}{c}-0,01467 \\ -1,01517 \\ 0 \\ 0\end{array}\right) y_{2}$.

Dabei bezeichnet die Variable $\mathrm{y}_{2}$ den Veränderungsbetrag des Kredits $\mathrm{B}_{2}$. Die Gleichung zeigt an, wie die A-Kredite (Variable $\mathrm{x}_{1}, \mathrm{x}_{2}, \mathrm{x}_{3}, \mathrm{x}_{4}$ ) zu verändern sind, um die Zahlungskonsequenzen einer Veränderung des Kredits $\mathrm{B}_{2}$ in den Jahren 2017 bis 2020 auszugleichen. Der maximale Reduktionsbetrag von Kredit $\mathrm{B}_{2}$ hängt von den einzelnen Variationsgrenzen der Variablen $\mathrm{x}_{1}, \mathrm{x}_{2}, \mathrm{x}_{3}$ und $\mathrm{x}_{4}$ sowie $\mathrm{y}_{2} \mathrm{ab}$. Er ist in $A b b .6$ berechnet. Danach liegen die Grenzen für die Ausgleichszahlungen der Standardfinanzierung durchweg höher als der bestehende Kreditbetrag von $\mathrm{B}_{2}$ selbst. Der Kredit $\mathrm{B}_{2}$ kann demnach vollständig zurückgezahlt werden.

Die Rückzahlung von $\mathrm{B}_{2}$ ist deshalb eine erste finanziell empfehlenswerte Umschuldungsmaßnahme. Sie hat zur

\begin{tabular}{|c|c|c|c|c|c|c|c|c|c|}
\hline \multirow{2}{*}{$\begin{array}{c}\text { Jahr } \\
\mathbf{t}\end{array}$} & \multirow{2}{*}{$\begin{array}{l}\text { Abzin- } \\
\text { sungs- } \\
\text { faktor }\end{array}$} & \multicolumn{2}{|c|}{ Anlage $B_{1}$} & \multicolumn{2}{|c|}{ Kredit $B_{2}$} & \multicolumn{2}{|c|}{ Kredit $B_{3}$} & \multicolumn{2}{|c|}{ Kredit $B_{4}$} \\
\hline & & Zeitwert & Barwert & Zeitwert & Barwert & Zeitwert & Barwert & Zeitwert & Barwert \\
\hline 0 & 1,00000 & $-1,00000$ & $-1,00000$ & 1,00000 & 1,00000 & 1,00000 & 1,00000 & 1,00000 & 1,00000 \\
\hline 1 & 0,96712 & 1,01900 & 0,98549 & $-0,07100$ & $-0,06867$ & $-0,37896$ & $-0,36650$ & $-0,07500$ & $-0,07253$ \\
\hline 2 & 0,89745 & & & $-1,07100$ & $-0,96117$ & $-0,37896$ & $-0,34010$ & $-0,37500$ & $-0,33654$ \\
\hline 3 & 0,82095 & & & & & $-0,37896$ & $-0,31111$ & $-0,35250$ & $-0,28939$ \\
\hline 4 & 0,68645 & & & & & & & $-0,43000$ & $-0,29518$ \\
\hline \multicolumn{2}{|c|}{ Kapitalwert } & \multicolumn{2}{|c|}{$-0,01451 €$} & \multicolumn{2}{|c|}{$-0,02983 €$} & \multicolumn{2}{|c|}{$-0,01772 €$} & \multicolumn{2}{|r|}{$0,00636 €$} \\
\hline
\end{tabular}
Folge, dass die Geschäfte der Standardfinanzierung in ihrer Höhe anzupassen sind. Die erforderlichen Änderungen berechnen sich nach der Gleichung

Abb. 5: Kapitalwerte der B-Finanzgeschäfte bei der Standardfinanzierung A 


\begin{tabular}{|c|c|c|c|c|c|c|c|c|c|c|}
\hline $\mathbf{i}$ & $B_{i}^{u}$ & $\leq$ & & $\mathbf{x}_{\mathbf{i}}$ & & $\leq$ & $\mathbf{B}_{i}^{\circ}$ & Ergebnis für neg & ativ & es $y_{2}$ \\
\hline$A_{1}$ & $-440.000 €$ & $\leq$ & $x_{1}=$ & $-0,01467$ & $y_{2}$ & $\leq$ & $560.000 €$ & $-38.180 .450 €$ & $\leq$ & $y_{2}$ \\
\hline$A_{2}$ & $-350.000 €$ & $\leq$ & $x_{2}=$ & $-1,01517$ & $\mathrm{y}_{2}$ & $\leq$ & $650.000 €$ & $-640.289 €$ & $\leq$ & $\mathrm{y}_{2}$ \\
\hline$A_{3}$ & $-660.000 €$ & $\leq$ & $x_{3}=$ & 0,00000 & $\mathrm{y}_{2}$ & $\leq$ & $800.000 €$ & & & \\
\hline$A_{4}$ & $-620.101 €$ & $\leq$ & $\mathrm{x}_{4}=$ & 0,00000 & $y_{2}$ & $\leq$ & $800.000 €$ & & & \\
\hline $\mathbf{B}_{2}$ & $-580.000 €$ & $\leq$ & & & $\mathrm{y}_{2}$ & $\leq$ & $0 €$ & $-580.000 €$ & $\leq$ & $\mathbf{y}_{2}$ \\
\hline
\end{tabular}

Abb. 6: Beschränkungen für die Rückzahlung von Kredit $B_{2}$

\begin{tabular}{|l|c|c|c|c|}
\hline Kredit & $\mathbf{A}_{1}$ & $\mathbf{A}_{\mathbf{2}}$ & $\mathbf{A}_{3}$ & $\mathbf{A}_{4}$ \\
\hline Untergrenze $\mathbf{B}_{\mathbf{i}}^{\mathbf{u}}$ & $-448.507 €$ & $-938.796 €$ & $-660.000 €$ & $-620.101 €$ \\
\hline Obergrenze $\mathbf{B}_{\mathbf{i}}^{\circ}$ & $551.493 €$ & $61.204 €$ & $800.000 €$ & $800.000 €$ \\
\hline
\end{tabular}

Abb. 7: Neue Variationsgrenzen nach Ablösung des Kredits $B_{2}$

\begin{tabular}{|c|c|c|c|c|c|c|c|c|c|c|}
\hline $\mathbf{i}$ & $B_{i}^{u}$ & $\leq$ & & $\mathbf{x}_{\mathbf{i}}$ & & $\leq$ & $\mathbf{B}_{i}^{\circ}$ & Ergebnis für ne & jati & es $y_{3}$ \\
\hline$A_{1}$ & $-448.507 €$ & $\leq$ & $x_{1}=$ & 0,03850 & $\mathrm{y}_{3}$ & $\leq$ & $551.493 €$ & $-11.650 .150 €$ & $\leq$ & $y_{3}$ \\
\hline$A_{2}$ & $-938.796 €$ & $\leq$ & $x_{2}=$ & 0,01937 & $y_{3}$ & $\leq$ & $61.204 €$ & $-48.464 .636 €$ & $\leq$ & $\mathrm{y}_{3}$ \\
\hline$A_{3}$ & $-660.000 €$ & $\leq$ & $x_{3}=$ & $-1,07559$ & $y_{3}$ & $\leq$ & $800.000 €$ & $-743.780 €$ & $\leq$ & $y_{3}$ \\
\hline $\mathbf{A}_{4}$ & $-620.101 €$ & $\leq$ & $x_{4}=$ & 0,00000 & $y_{3}$ & $\leq$ & $800.000 €$ & & & \\
\hline $\mathrm{B}_{3}$ & $-1.200 .000 €$ & $\leq$ & & & $\mathrm{y}_{3}$ & $\leq$ & unbegrenzt & $-1.200 .000 €$ & $\leq$ & $y_{3}$ \\
\hline
\end{tabular}

Abb. 8: Beschränkungen für die Rückzahlung von Kredit $B_{3}$

$$
\left(\begin{array}{l}
x_{1} \\
x_{2} \\
x_{3} \\
x_{4}
\end{array}\right)=\left(\begin{array}{c}
-0,01467 \\
-1,01517 \\
0 \\
0
\end{array}\right) \cdot(-580.000)=\left(\begin{array}{c}
8.507 \\
588.796 \\
0 \\
0
\end{array}\right) \text {. }
$$

Zur Ablösung des Kredits $B_{2}$ ist also der Kredit $A_{1}$ um $8.507 €$ und der Kredit $A_{2}$ um $588.796 €$ zu erhöhen. Bei gleichbleibenden Zahlungsverpflichtungen in den Folgeperioden stehen der Blitz AG im Ergebnis somit heute $17.303 €$ mehr zur Verfügung; dies ist der Arbitragegewinn dieser Umschuldungsmaßnahme. Durch die Anpassung der A-Kredite ergeben sich neue Variationsgrenzen für die Standardfinanzierung. Diese sind in $A b b .7$ angegeben.

Nach der ersten Umschuldungsmaßnahme ist damit Kredit $\mathrm{B}_{2}$ ausgeschieden. Da die Standardfinanzierung dadurch nicht geändert wurde, gelten die Kapitalwerte der Abb. 5 nach wie vor. Danach ist Kredit $B_{3}$ das nächste zu betrachtenden Finanzierungsgeschäft. Es handelt sich wieder um einen zu reduzierenden Kredit. Zur Reduktion von Kredit $\mathrm{B}_{3}$ um den Betrag $\mathrm{y}_{3}$ sind folgende Anpassungen der Standardfinanzgeschäfte erforderlich:

$\left(\begin{array}{l}x_{1} \\ x_{2} \\ x_{3} \\ x_{4}\end{array}\right)=-\left(\begin{array}{cccc}0,96712 & -0,05042 & -1,01829 & 0,10159 \\ 0 & 0,94787 & -0,99898 & 0,05111 \\ 0 & 0 & 2,83822 & -2,83822 \\ 0 & 0 & 0 & 3,37197\end{array}\right) \cdot\left(\begin{array}{c}0,37896 \\ 0,37896 \\ 0,37896 \\ 0\end{array}\right) y_{3}=\left(\begin{array}{c}0,03850 \\ 0,01937 \\ -1,07559 \\ 0\end{array}\right) y_{3}$.
Aus der Berechnung der potenziellen Umschuldungsgrenzen in $A b b .8$ ergibt sich, dass der Kredit $\mathrm{B}_{3}$ um maximal $743.780 €$ reduziert werden kann. Eine weitergehende Reduktion von Kredit $B_{3}$ scheitert daran, dass die obere Intervallgrenze von Kredit $A_{3}$ erreicht ist.

Die Änderungen in der Standardfinanzierung berechnen sich zu:

$\left(\begin{array}{l}x_{1} \\ x_{2} \\ x_{3} \\ x_{4}\end{array}\right)=\left(\begin{array}{c}0,03850 \\ 0,01937 \\ -1,07559 \\ 0\end{array}\right) \cdot(-743.780)=\left(\begin{array}{c}-28.634 \\ -14.408 \\ 800.000 \\ 0\end{array}\right)$.

Der Kredit $A_{1}$ wird um $28.634 €$, der Kredit $A_{2}$ um $14.408 €$ reduziert. Der Kredit $A_{3}$ wird um den Maximalbetrag von $800.000 €$ erhöht. Der Arbitragegewinn der Umschuldungsmaßnahmen beläuft sich auf $13.178 €$. Da der Kredit $\mathrm{A}_{3}$ nun völlig ausgeschöpft ist, kann er nicht mehr in beiden Richtungen variiert werden. Er scheidet damit aus der Standardfinanzierung aus. An seine Stelle tritt nun Kredit $B_{3}$. Da jener einerseits nicht völlig zurückgezahlt ist, andererseits aber auch noch erhöht werden kann, steht er für weitere Finanzierungen zur Verfügung. Nach den bisherigen Ergebnissen ist er zwar finanziell ungünstiger als Kredit $A_{3}$. Da jener aber bereits maximal in Anspruch genommen wurde, rückt nun $\mathrm{B}_{3}$ für künftige Finanzierungen nach. Die aktualisierten Intervallgrenzen sind in $A b b .9$ aufgeführt. 


\begin{tabular}{|l|c|c|c|c|}
\hline Kredit & $\mathbf{A}_{\mathbf{1}}$ & $\mathbf{A}_{\mathbf{2}}$ & $\mathbf{B}_{3}$ & $\mathbf{A}_{\mathbf{4}}$ \\
\hline Untergrenze $\mathbf{B}_{\mathbf{i}}^{\mathbf{u}}$ & $-419.873 €$ & $-924.389 €$ & $-456.220 €$ & $-620.101 €$ \\
\hline Obergrenze $\mathbf{B}_{\mathbf{i}}^{\circ}$ & $580.127 €$ & $75.611 €$ & $743.780 €$ & $800.000 €$ \\
\hline
\end{tabular}

Abb. 9: Variationsgrenzen nach der zweiten Umschuldung

\begin{tabular}{|l|c|c|c|c|}
\hline Kredit & $\mathbf{A}_{\mathbf{1}}$ & $\mathbf{A}_{\mathbf{2}}$ & \multicolumn{1}{c|}{$\mathbf{B}_{3}$} & $\mathbf{A}_{\mathbf{4}}$ \\
\hline Untergrenze $\mathbf{B}_{\mathbf{i}}^{\mathbf{u}}$ & $-345.961 €$ & $-924.389 €$ & $-456.220 €$ & $-620.101 €$ \\
\hline Obergrenze $\mathbf{B}_{\mathbf{i}}^{\circ}$ & $654.039 €$ & $75.611 €$ & $743.780 €$ & $800.000 €$ \\
\hline
\end{tabular}

Abb. 10: Variationsgrenzen nach der dritten Umschuldung

\begin{tabular}{|l|c|c|c|c|}
\hline Kredit & $\mathbf{A}_{\mathbf{1}}$ & $\mathbf{A}_{\mathbf{2}}$ & $\mathbf{B}_{\mathbf{3}}$ & $\mathbf{B}_{\mathbf{4}}$ \\
\hline Untergrenze $\mathbf{B}_{\mathbf{i}}^{\mathbf{u}}$ & $-461.222 €$ & $-915.268 €$ & $-543.680 €$ & $-1.277 .671 €$ \\
\hline Obergrenze $\mathbf{B}_{\mathbf{i}}^{\circ}$ & $538.778 €$ & $84.732 €$ & $656.320 €$ & $172.329 €$ \\
\hline
\end{tabular}

Abb. 1l: Variationsgrenzen der neuen Standardfinanzierung

Da sich durch die zweite Umschuldungsmaßnahme die Zusammensetzung der Standardfinanzierung geändert hat, sind neue Abzinsungsfaktoren für die jetzt gültige Finanzierung zu bestimmen. Die Matrix A der Rückzahlungskoeffizienten und ihre Inverse sehen jetzt wie folgt aus:

$A=\left(\begin{array}{cccc}1,03400 & 0,05500 & 0,37896 & 0,29656 \\ 0 & 1,05500 & 0,37896 & 0,29656 \\ 0 & 0 & 0,37896 & 0,29656 \\ 0 & 0 & 0 & 0,29656\end{array}\right)$

$A^{-1}=\left(\begin{array}{cccr}0,96712 & -0,05042 & -0,91670 & 0,00000 \\ 0 & 0,94787 & -0,94787 & 0,00000 \\ 0 & 0 & 2,63877 & -2,63877 \\ 0 & 0 & 0 & 3,37197\end{array}\right)$

Multipliziert man $\mathrm{A}^{-1}$ mit dem (neuen) Barzahlungsvektor $\mathrm{a}^{(0)^{2}}=(1,1,1,1)$, so erhält man die aktuellen Abzinsungsfaktoren $0,96712,0,89745,0,77420$ und 0,73321 für die Jahre 2017 bis 2020. Mit ihnen lassen sich analog zu $A b b .5$ die jetzt geltenden Kapitalwerte der verbleibenden B-Finanzgeschäfte $\mathrm{B}_{1}$ und $\mathrm{B}_{4}$ berechnen. Für Finanzanlage $B_{1}$ erhält man einen Wert von $-0,01451 €$, für Kredit $B_{4}$ von $+0,00274 €$. Damit ist es zunächst vorteilhaft, die Geldanlage $B_{1}$ aufzulösen. Bei dem bestehenden Kreditzins für die einjährige Finanzierung lohnt sich eine gleichzeitige einjährige Geldanlage nicht - in diesem Fall ein auch unmittelbar erkennbarer Sachverhalt. Die für einen Reduktionsbetrag der Geldanlage $\mathrm{B}_{1}$ von $\mathrm{y}_{1}$ erforderlichen Anpassungen der Standardfinanzierung berechnen sich zu

$\left(\begin{array}{l}x_{1} \\ x_{2} \\ y_{3} \\ x_{4}\end{array}\right)=-\left(\begin{array}{ccrr}0,96712 & -0,05042 & -0,91670 & 0,00000 \\ 0 & 0,94787 & -0,94787 & 0,00000 \\ 0 & 0 & 2,63877 & -2,63877 \\ 0 & 0 & 0 & 3,37197\end{array}\right) \cdot\left(\begin{array}{c}-1,01900 \\ 0 \\ 0 \\ 0\end{array}\right) y_{1}=\left(\begin{array}{c}0,98549 \\ 0 \\ 0 \\ 0\end{array}\right) y_{1}$.

Eine Reduktion der Anlage $B_{1}$ um einen Euro erlaubt folglich, zum Ausgleich den Kredit $A_{1}$ um 0,98549€ zu tilgen.
Pro Euro entsteht also ein Gewinn von fast 1,5 Cent. Die maximal mögliche Reduktion von Anlage $B_{1}$ ist lediglich vom derzeitigen Anlagebetrag von $B_{1}$ selbst (75.000 €) sowie von der bestehenden Kredithöhe von $A_{1}(419.873 €)$ abhängig. Somit gilt $\mathrm{y}_{1} \geq-75.000 €$ und entsprechend $0,98549 \mathrm{y}_{1} \geq-419.873 €$ bzw. $\mathrm{y}_{1} \geq-426.054 €$. Die Begrenzung des Guthabenbetrags von $\mathrm{B}_{1}$ wirkt stärker. Daher wird die maximal mögliche Reduktion von $\mathrm{B}_{1}$ erreicht, indem diese Geldanlage vollständig abgebaut wird. Im Gegenzug wird der Kredit $A_{1}$ um $73.912 €$ reduziert. Der Arbitragegewinn beträgt $1.088 €$. Die neuen Intervallgrenzen sind $A b b .10 \mathrm{zu}$ entnehmen.

Nach dieser Umschuldungsmaßnahme verbleibt nur Kredit $\mathrm{B}_{4}$ als ,„überzähliges“ Finanzgeschäft. Sein Kapitalwert von $+0,00274 €$ zeigt, dass es vorteilhaft ist, ihn auszuweiten. Allerdings sind hier die damit verbundenen Umschuldungsmaßnahmen weniger leicht ohne Rechnung erkennbar als im vorigen Fall. Vielmehr sind, wie das Durchlaufen der Umschuldungsschritte im Einzelnen ergibt, mit der angestrebten Erhöhung des Kredits $\mathrm{B}_{4}$ gleichzeitig auch die Kredite $\mathrm{A}_{1}$ und $\mathrm{B}_{3} \mathrm{zu}$ erhöhen. Damit gelingt es dann, die Kredite $\mathrm{A}_{2}$ und $\mathrm{A}_{4}$ zu reduzieren. All dies ergibt sich rechnerisch auf die gleiche Weise wie in den bisherigen drei Umschuldungsschritten. Im Ergebnis wird Kredit $B_{4}$ um $427.671 €$ erhöht, Kredit $A_{1}$ um $115.262 €$ und Kredit $B_{3}$ um 87.461 €. Gleichzeitig können die Kredite $\mathrm{A}_{2}$ und $\mathrm{A}_{4}$ um $9.121 €$ bzw. um $620.101 €$ reduziert werden. Eine weitere Ausweitung von Kredit $B_{4}$ ist nicht möglich, da der ungünstigere Kredit $A_{4}$ nun bereits vollständig zurückgezahlt ist. Er scheidet folglich auch aus der Standardfinanzierung aus. Hier wird er durch Kredit $\mathrm{B}_{4}$ ersetzt. Der Arbitragegewinn der Maßnahmen beträgt $1.171 €$.

Nach dieser letzten Umschuldungsmaßnahme steht die Standardfinanzierung, mit der neue Projekte beurteilt werden können, eindeutig fest: Sie besteht aus den Krediten $A_{1}, A_{2}, B_{3}$ und $B_{4}$. Sie können, wie die Neuberechnung 
der Intervallgrenzen nach der letzten Umschuldung ergibt, innerhalb der in $A b b .11$ angegebenen Grenzen in Anspruch genommen werden.

Es ist also bei jedem dieser vier Kredite sowohl eine Erhöhung (bis zur Obergrenze $\mathrm{B}_{\mathrm{i}}^{\circ}$ ) als auch eine Reduzierung (bis zur Untergrenze $\mathrm{B}_{\mathrm{i}}^{\mathrm{u}}$ ) möglich.

\section{Aufgabe 2c)}

Die Summe der Arbitragegewinne aus den vier Umschuldungsmaßnahmen beträgt $32.740 €$.

\section{Alle wichtigen Steuergesetze in einem Band.}

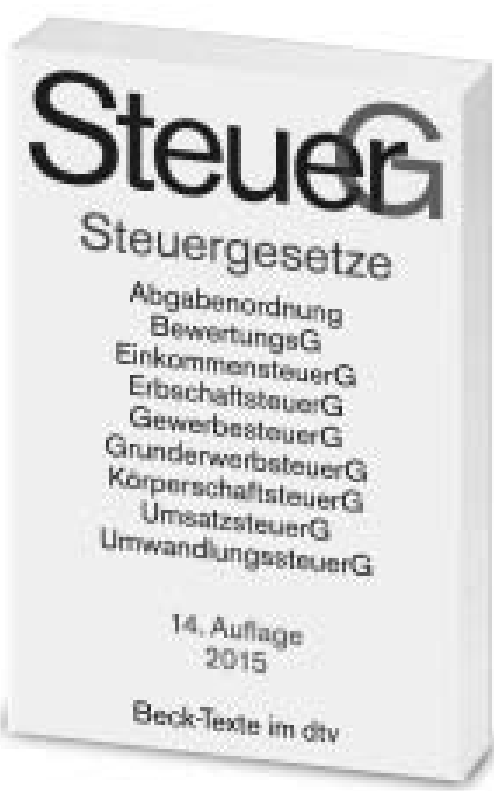

Textausgabe mit ausführlichem Sachverzeichnis.

14. Auflage. 2015. Stand: 15. September 2015. XII, 1546 Seiten. Kartoniert $€ 9,90$ (dtv-Band 5765)

\section{Alles in Einem}

Alle wichtigen Steuergesetze, die in der Praxis und in der Ausbildung ständig gebraucht werden, in einem Band mit vielen redaktionellen Hinweisen - praktisch, handlich, immer griffbereit.

\section{Mit Stand 15. September 2015}

Neben Auszügen aus dem AEUV, FVG, GG, GKG und dem HGB wurde diese Ausgabe jetzt um das VermBG mit DV und das WoPG erweitert.

\section{Täglich nützlich}

für Praktiker (RA, StB, FAStR), Auszubildende in steuerberatenden Berufen, Studenten an Universitäten Fachhochschulen und Akademien, Finanzverwaltungen sowie Mitarbeiter in Steuerabteilungen. 


\section{Aktuelle Wirtschaftsgesetze 2016.}

\section{Diese bewährte Textausgabe}

enthält in einem Band alle im Wirtschaftsleben relevanten Rechtsvorschriften:

\section{Allgemeines Zivilrecht}

- Bürgerliches Gesetzbuch (Auszug: Allgemeiner Teil, Recht der Schuldverhältnisse, Sachenrecht)

- Einführungsgesetz zum Bürgerlichen Gesetzbuch

- Allgemeines Gleichbehandlungsgesetz

- Produkthaftungsgesetz

Handelsrecht

- Handelsgesetzbuch (ohne Seehandelsrecht)

- UN-Kaufrecht (CISG)

\section{Wettbewerbsrecht}

- Gesetz gegen den unlauteren Wettbewerb (UWG)

- Preisangabenverordnung

Gesellschaftsrecht

- Aktiengesetz

- GmbH-Gesetz

- Genossenschaftsgesetz

- Partnerschaftsgesellschaftsgesetz

- Umwandlungsgesetz

Insolvenzrecht

- Insolvenzordnung

\section{Kapitalmarktrecht}

- Kreditwesengesetz

- Wertpapierhandelsgesetz

\section{Die Neuauflage}

enthält insbesondere die Änderungen durch das Bilanzrichtlinie-Umsetzungsgesetz (BilRUG), das Kleinanlegerschutzgesetz, das Gesetz zur Verringerung der Abhängig-

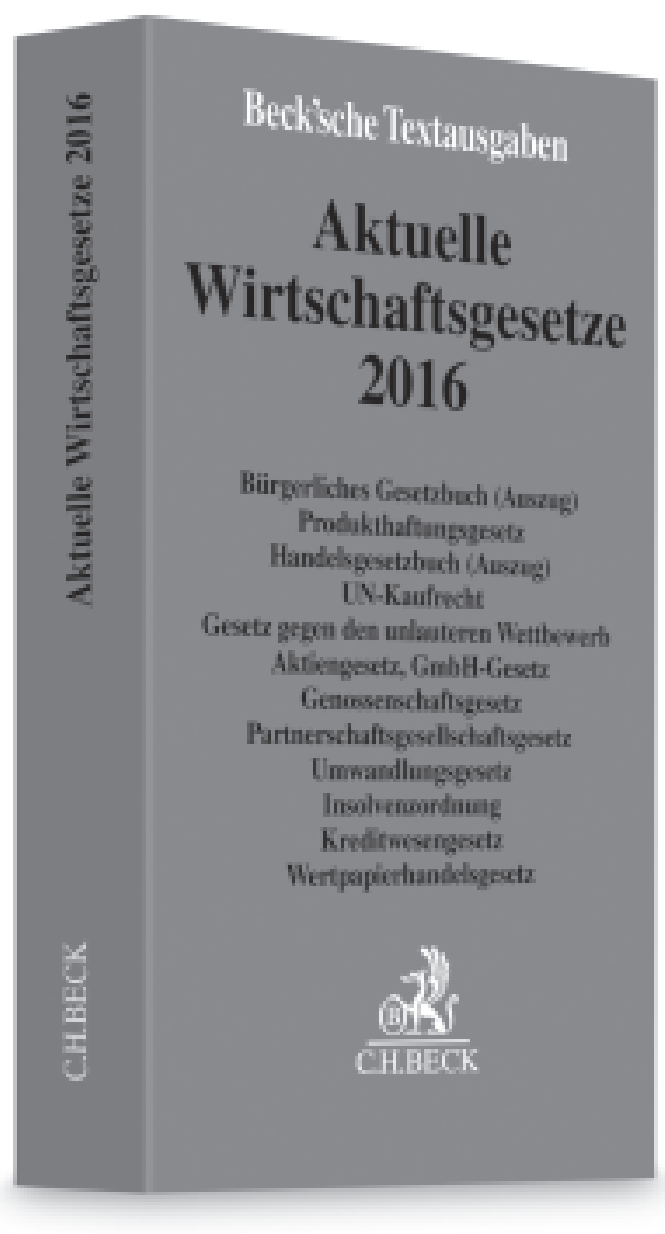

Aktuelle Wirtschaftsgesetze 2016

17. Auflage. 2016. VIII, 1538 Seiten.

Kartoniert $€$ 9,80

ISBN 978-3-406-68141-7

Mehr Informationen:

www.beck-shop.de/bglvlt

keit von Ratings und durch das BRRD-Umsetzungsgesetz und das DGSD-Umsetzungsgesetz. 


\section{Der Leitfaden durch das europäische Steuerrecht.}

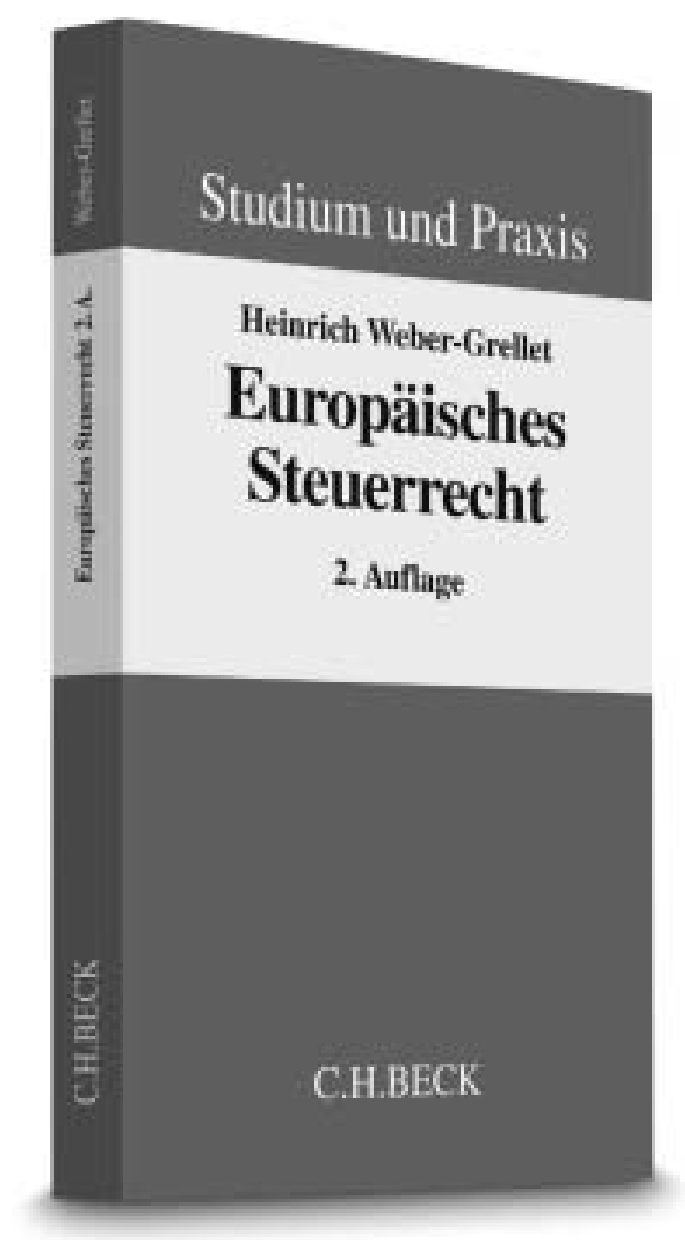

Weber-Grellet Europäisches Steuerrecht

2. Auflage. 2016. XXIII, 233 Seiten. Kartoniert $€ 34,90$

ISBN 978-3-406-68011-3

Neu im April 2016

Mehr Informationen: www.beck-shop.de/bgjice

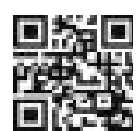

\section{Steuern im Spannungsfeld}

Rechtsetzungsbedürfnisse auf europäischer Ebene einerseits und die Wahrung der Steuerautonomie auf nationaler Ebene andererseits machen das Steuerrecht zu einem interessanten Spannungsfeld innerhalb der europäischen Integration. Der renommierte Schmidt-Autor und Vorsitzender Richter am BFH a.D. Prof. Dr. habil. Heinrich Weber-Grellet gibt in diesem Band einen kompakten Überblick über den aktuellen Diskussionsstand. Didaktisch aufgebaut liefert das Werk klare Argumentationshilfen für und wider die Europarechtskonformität einzelner Vorschriften.

\section{Der Inhalt:}

- Grundlagen des Europäischen Steuerrechts

- Direkte Harmonisierung indirekter Steuern

- Indirekte Harmonisierung der direkten Steuern

- Anwendung und Durchsetzung des EU-Rechts

- Konkrete Auswirkungen des EU-Rechts auf das deutsche Steuerrecht

\section{Die Neuauflage}

Aufgegriffen werden alle seit der ersten Auflage ergangenen Urteile des EuGH sowie alle nationalen Umsetzungen europäischer Vorgaben in nationales Recht. Am Ende jedes Kapitels befindet sich nunmehr jeweils eine Zusammenfassung mit den wichtigsten Erkenntnissen.

"Ein lesenswertes Buch, das für Studium und Praxis gleichermaßen empfohlen werden kann."

Priv.-Doz. Dr. Carl-Heinz Witt, LL.M., in: Zeitschrift für Gemeinschaftsprivatrecht, 04/2006, zur 1. Auflage 2005 


\section{Das neue revolutionäre Management-System.}

\section{Holacracy}

ist eine der faszinierendsten Managementphilosophien seit Six Sigma. Doch anders als Six Sigma und weitere autoritäre, Top-down orientierte Strukturen, versetzt Holakratie jeden Mitarbeiter in einer Organisation in die Lage, nach bestimmten Prinzipien selbstständig Entscheidungen zu treffen. Das neue Buch zeigt die Praktiken auf, holakratische Strukturen unternehmensweit oder in ausgewählten Abteilungen zu implementieren.

\section{Brian Robertson}

ist US-amerikanischer Unternehmer. Er gründete in den 1990er Jahren »Ternary Software«. Hier beschäftigte er sich zum ersten Mal intensiv mit alternativen Organisationsformen. Nach dem Verkauf des Unternehmens startete er mit "HolacracyOne" ein Beratungsunternehmen, das Organisationen hilft, eine holakratische Kultur zu implementieren.

\section{Ein Muss}

für Unternehmer, Organisationsentwickler und Unternehmensberater.

\section{"Ein elegantes und ineinandergreifendes System von} Praktiken der Selbstführung."

Frederic Laloux

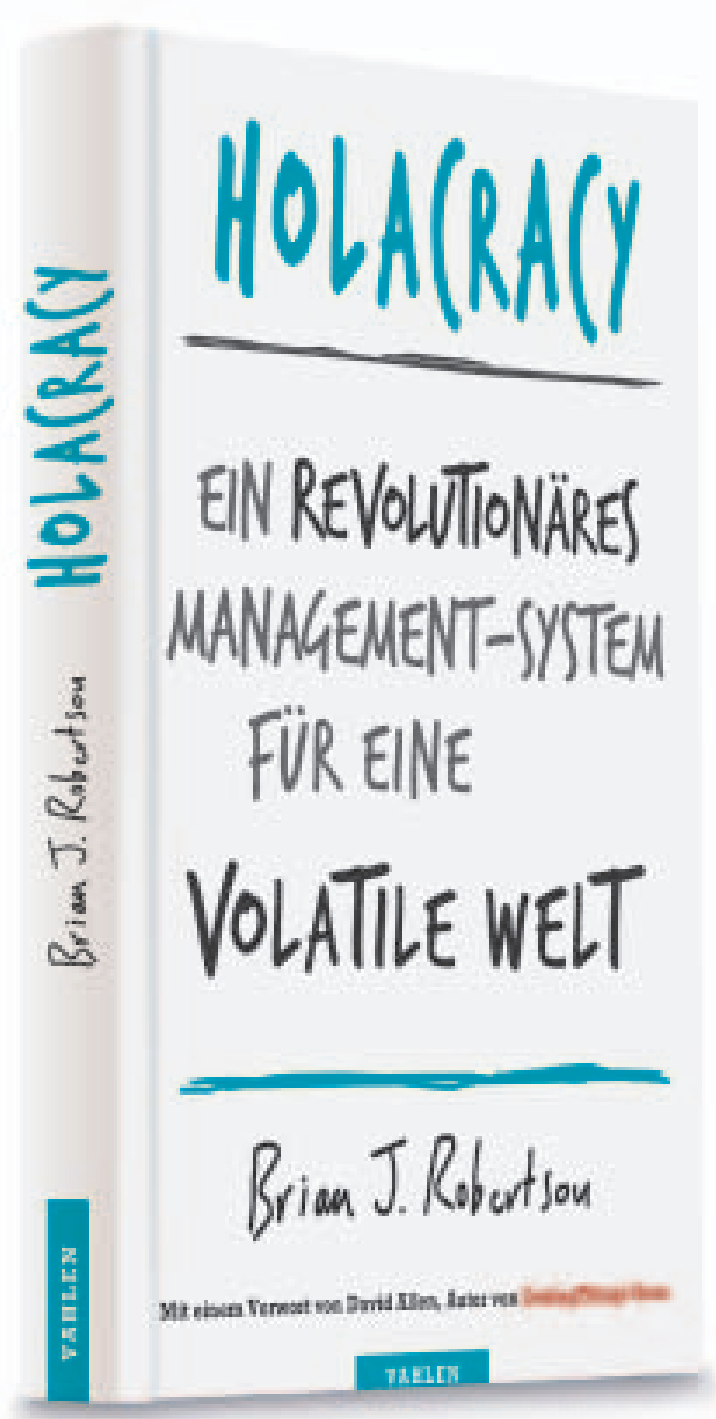

Robertson

Holacracy

2016. XV, 205 Seiten.

Gebunden $€ 24,90$

ISBN 978-3-8006-5087-3

Neu im März 2016

Portofrei geliefert:

vahlen.de/15478815 


\section{Das Handbuch zur Kosten- und Erlösrechnung.}

\section{Dieses Standardwerk}

liefert einen umfassenden Überblick über die Aufgaben, Techniken und Systeme der Kosten- und Erlösrechnung. Zunächst führt es in die Grundlagen ermittlungsorientierter Systeme ein. Dazu gehören die Kostenarten-, Kostenstellen- und Kostenträgerrechnung.

Daran schließt sich die Darstellung planungs- und verhaltenssteuerungsorientierter Systeme an. Dabei handelt es sich um Methoden wie Prozesskosten-, Grenzplankosten- oder Deckungsbeitragsrechnungen und Target Costing, die im Alltag von höchster praktischer Relevanz sind.

Abgeschlossen wird das Buch durch die Behandlung aktueller Weiterentwicklungen auf dem Gebiet der Kostenrechnung. Hierbei spielen insbesondere die Herausforderungen der Preisregulierung bei den Strom-, Gas- und Telekommunikationsmärkten eine große Rolle.

„Das Werk bietet Studierenden und Praktikern den State of the Art einer praxisorientierten Theorie der Kostenund Erlösrechnung (...) Diese Neuauflage zählt zu den Spitzentiteln der Kostenrechnung."

In Zeitschrift Controller Magazin zur 9. Auflage

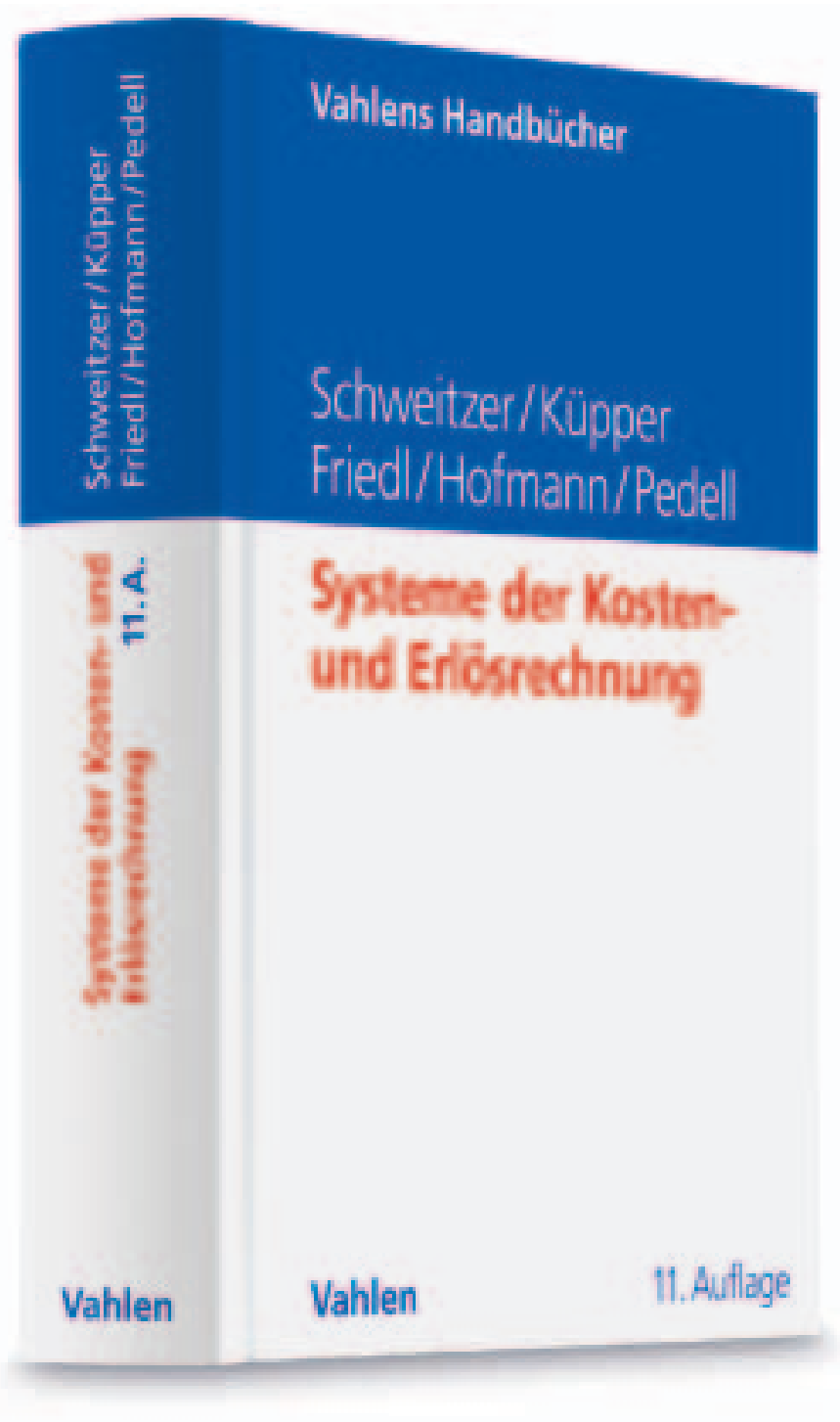

Schweitzer/Küpper/Friedl/ Hofmann/Pedell

Systeme der Kosten- und Erlösrechnung

11. Auflage. 2016. XVI, 907 Seiten.

Gebunden $€ 49,80$

ISBN 978-3-8006-5027-9

Portofrei geliefert:

vahlen.de/14833866 